\title{
Significance of ST Segment Elevation in Lead aVR in Patients with Non-ST Elevation Acute Coronary Syndrome
}

\author{
PRITAM KUMAR GACHCHHADAR, MANZOOR MAHMOOD, DIPAL KRISHNA ADHIKARY, \\ MSI TIPU CHOWDHURY, MD. ASHRAF UDDIN SULTAN, MRM MANDAL, \\ KHALED MD. IQBAL, SAJAL KRISHNA BANERJEE
}

Department of Cardiology, Bangabandhu Sheikh Mujib Medical University (BSMMU), Dhaka

Address of Correspondence: Dr. Pritam Kumar Gachchhadar, Resident, Department of Cardiology, Bangabandhu Sheikh Mujib Medical University (BSMMU), Dhaka. E mail: pritam.gachchhadar@gmail.com

\begin{abstract}
:
Background: As acute occlusion of the left main (LM) artery causes life-threatening hemodynamic deterioration and malignant arrhythmias, resulting in an adverse outcome, a rapid diagnosis and subsequent urgent revascularization with percutaneous coronary intervention (PCI) or coronary bypass surgery is very important in this subset of patients. The 12-lead electrocardiogram (ECG) is a crucial tool in the diagnosis and risk stratification of acute coronary syndrome (ACS). Unlike other 11 leads, lead aVR has been long neglected until recent years.

Objective: To determine the accuracy of 12-lead electrocardiography in predicting left main and/or triplevessel disease in patients with non-ST elevation acute coronary syndrome (NSTE-ACS).

Methodology: This cross sectional observational study carried out among patients presenting with

non-ST elevation acute coronary syndrome at Cardiac Emergency Department or CCU of BSMMU. This study was conducted from May 2017 to April 2018. A total of 36 patients meeting the eligibility criteria were consecutively included. Data collection was carried out by using a questionnaire. Informed written consent was obtained from the hospital authority. Analysis of data was finally done with Statistical Package for Social Science program 17 version of computer on the basis of different variables.

Result: As ST-segment elevation in lead aVR is a continuous variable, a suitable cut-offfor ST-elevation in lead aVR was found out for diagnosing LM and/or triple vessel disease (TVD) using ROC curve. The cut-off value was $0.75 \mathrm{~mm}$ which gave us an optimum sensitivity of $88.5 \%$ and a specificity of $80 \%$ with an area under the curve being $0.892(95 \% C I=0.785-1.000)$, $p<0.001$. The area under the curve demonstrated that $89.2 \%$ of the LM and/or TVD were correctly diagnosed with ST elevation e” $0.75 \mathrm{~mm}$ in lead aVR in patients with non-ST segment elevation acute coronary syndrome. The positive predictive value was commendably high (92\%) and negative predictive value was no less (72.7\%) with an overall diagnostic accuracy of $86 \%$. Conclusion: From the findings of the study it can be concluded that ST-segment elevation e" $0.75 \mathrm{~mm}$ in lead aVR in patients of non-ST segment elevation acute coronary syndrome had optimum sensitivity and specificity with an appreciably high overall diagnostic accuracy. The ST-segment elevation e”0.75 mm in lead aVR in patients with non-STE-ACS can differentiate LM and/or triple vessel disease with fair degree of accuracy.
\end{abstract}

Key wards: Non-ST elevation acute coronary syndrome (NSTE-ACS), Triple-vessel disease (TVD).

University Heart Journal 2018; 14(2): 71-76

Introduction:

Acute coronary syndrome remains the leading cause of morbidity and mortality worldwide. It will continue to rise as the prevalence of patients with obesity and diabetes increases. ${ }^{1}$ Patients with non-ST elevation acute myocardial infarction (AMI) are heterogeneous with respect to the pathophysiological mechanisms, the size of the infarction, and the amount of jeopardized myocardium. Early risk stratification is a fundamental step in the management of this condition. ${ }^{2}$

Presentation of ACS is different depending on the coronary artery involvement, severity, degree of collateral circulation and myocardial oxygen demand. Critical stenosis in the proximal part of the left anterior descending, severe three vessel disease and left main stem stenosis have all been recognized as 
clinicalconditions complicated by a high incidence of large infarction, pump failure, arrhythmias and sudden death. As because many effective treatment modes are available currently, early recognition of these circumstances is crucial for appropriate management. ${ }^{3}$

Significant narrowing of the main coronary arteries carries high risks for the patients, since occlusion of these vessels if unprotected by collateral flow or a patients bypass grafts to either the left anterior descending (LAD) or left circumflex (LCX) artery compromise flow to approximately $75 \%$ of the left ventricle. Thus, prediction of left main coronary artery (LMCA) obstruction is important with regard to selecting appropriate treatment strategy and estimating prognosis3.

The 12-leads electrocardiogram (ECG) is a crucial tool in the diagnosis and risk stratification of acute coronary syndrome (ACS). ST-segment elevation in lead aVR predicts LM or three vessels disease in patients with nonST-segment elevation acute coronary syndromes. Unlike other 11 leads, lead aVR has been long neglected until recent years. However, recent investigations have shown that an analysis of ST-segment shift in lead aVR provides useful information on the coronary angiographic anatomy and risk stratification in ACS4. The purpose of the present study was to assess the independent predictive value of ST-segment elevation in lead aVR for LM/3VD in patients with non-ST-segment elevation acute coronary syndrome (NSTE-ACS).

\section{Materials and Methods:}

Study design: Cross sectional obsevational study

Study place: The study was carried out in the Department of Cardiology, University Cardiac Centre, BSMMU, Dhaka over a period of 12 months from May

2017 to April 2018.

Study population: Patients presenting with non-ST elevation acute coronary syndrome at Cardiac Emergency Department or CCU of BSMMU were the study population.

The eligibility criteria was as follows:

\section{Inclusion criteria:}

Patients having ST elevation in lead aVR with following criteria were included:

- Typical chest pain attributed to cardiac ischemia lasting at least $20 \mathrm{~min}$ and involving an unstable pattern of pain, including rest pain, new onset, severe or frequent angina (accelerating angina).
- Patients undergoing CAG during hospitalization before coronary intervention.

- Patients with recent NSTE-ACS

Exclusion criteria: Patients with the following criteria were excluded from the study:

- Conditions precluding the evaluation of ST segment on the ECG (LBBB, RBBB, left ventricular hypertrophy, ventricular pacing, ventricular preexcitation, non-ischemic cardiomyopathy, or antiarrhythmic drugs).

- Transient or persistent ST segment elevation in leads other than aVR.

- Q-wave acute MI on presentation.

- Recent $[<6$ months] PCI or Previous CABG.

The patients with non-ST elevation acute coronary syndrome attending at UCC, BSMMU were selected as case on the basis of predefined eligibility criteria (inclusion and exclusion criteria). Consecutive sampling technique was used to include the required number of patients who fulfilled the selection criteria. These patients were evaluated and managed according to the guidelinebased protocol of UCC.

Demographic data of the patients were collected and ECG were evaluated for ST-elevation in Lead aVR. Then CAG was done according to guideline protocol and patients' wish using SIEMENS AXIOM Artis by percutaneous femoral approach. Angiographic coronary lesion was evaluated by two independent observers. Patients with $50 \%$ or more stenosis in left main or $70 \%$ or more stenosis in all three coronary vessels (RCA, LAD and LCx) were included in one group and other patients with normal coronaries or insignificant stenosis significant stenosis in one or two vessels were included in another group.

Data were recorded in a pre-designed format including history, clinical examination, investigations, TTDE, and coronary angiogram. After completion of the data collection, the data were processed and analysed using SPSS (Statistical Package for Social Sciences), version 17.

\section{Results:}

Nearly three-quarters (72.2\%) of the patients were upper middle aged or elderly (50 or $>50$ years old), $22.2 \%$ middle aged ( $41-50$ years) and only $5.6 \%$ lower middle aged (d" 40 years). The mean age of the patients was 56.4 years with youngest and oldest patients being 34 and 75 years respectively. Patients were predominantly male 
$83.3 \%$ ) with male to female ratio being roughly $5: 1$ (Table I).

More than $55 \%$ of the patients were smoker $(27.8 \%$ current and another $27.8 \%$ previous smoker). Nearly $60 \%$ of the patients were hypertensive and $47.2 \%$ diabetic. Dyslipidaemia and family history of CAD were reported to be 27.8 and $33.3 \%$ respectively (Table II).

Table-I

Distribution of patients by their demographic characteristics $(n=36)$

\begin{tabular}{lcc}
\hline $\begin{array}{l}\text { Demographic } \\
\text { characteristics }\end{array}$ & Frequency & Percentage \\
\hline Age (years) & 02 & 5.6 \\
$\leq 40$ & 08 & 22.2 \\
$41-50$ & 26 & 72.2 \\
$>50$ & 30 & 83.3 \\
SexMale & 06 & 16.7 \\
\hline
\end{tabular}

$*$ Mean $=56.4 \pm 9.4$ years; Range $=34-75$ years.

Table II

Distribution of patients by their risk factors $(n=36)$

\begin{tabular}{lcc}
\hline Risk factors & Frequency & Percentage \\
\hline Smoking habit & & \\
Current smoker & 10 & 27.8 \\
Previous smoker & 10 & 27.8 \\
Non-smoker & 16 & 44.4 \\
Hypertension & 21 & 58.3 \\
Diabetes & 17 & 47.2 \\
Dyslipidaemia & 10 & 27.8 \\
F/H of CAD & 12 & 33.3 \\
\hline
\end{tabular}

Only $2(5.6 \%)$ patients had raised serum creatinine (serum creatinine $>1.5 \mathrm{mg} / \mathrm{dl}$ ). Elevated serum cholesterol (serum TC $>200 \mathrm{mg} / \mathrm{dl}$ ), elevated serum LDL (serum LDL $>130 \mathrm{mg} / \mathrm{dl}$ ) and raised serum Triglycerides (serum $\mathrm{Tg}>150 \mathrm{mg} / \mathrm{dl}$ ) were 27.8, 33.3 and $30.6 \%$ respectively, while low HDL was $58.3 \%$ (Table III).

Significant stenosis in left main (LM) and in major coronary arteries are shown in Table V. Eight patients (22.2\%) had significant LM disease. More than $90 \%$ had significant stenosis in LAD, $77.8 \%$ had significant stenosis in LCx and $72.2 \%$ had stenosis in RCA (Table IV).
Table-III

Distribution of patients by their

\begin{tabular}{lcc}
\hline $\begin{array}{l}\text { Laboratory } \\
\text { investigations }\end{array}$ & $\begin{array}{c}\text { Frequency } \\
(\%)\end{array}$ & $\begin{array}{c}\text { Mean } \pm \text { SD } \\
(\text { Range })\end{array}$ \\
\hline Serum creatinine & $02(5.6)$ & $1.2 \pm 0.8$ \\
$>1.5 \mathrm{mg} / \mathrm{dl}$ & & $0.54-6.02$ \\
Serum TC $>200 \mathrm{mg} / \mathrm{dl}$ & $10(27.8)$ & - \\
LDL $>130 \mathrm{mg} / \mathrm{dl}$ & $12(33.3)$ & - \\
Low HDL $($ with & $21(58.3)$ & - \\
respect to sex $)$ & $11(30.6)$ & - \\
TG $>150 \mathrm{mg} / \mathrm{dl}$ & - & $3.8 \pm 1.1$ \\
Troponin I (ng/dl) & & $(0.001-42.2)$ \\
& - & $57.4 \pm 9.8$ \\
CK-MB $(\mathrm{IU} / \mathrm{L})$ & & $(6-323)$ \\
\hline
\end{tabular}

Table-IV

Significant stenosis in LM/major coronary arteries $(n=36)$

\begin{tabular}{lcc}
\hline Significantstenosis & Frequency & Percentage \\
\hline LM $(\geq 50 \%)$ & 08 & 22.2 \\
LAD $(\geq 70 \%)$ & 33 & 91.7 \\
LCX $(\geq 70 \%)$ & 28 & 77.8 \\
RCA $(\geq 70 \%)$ & 26 & 72.2 \\
\hline
\end{tabular}

Two-thirds (66.7\%) of the patients had triple vessel disease (TVD), 11.1\% double vessel disease (DVD), 19.4\% single vessel disease (SVD) (Figure 1).

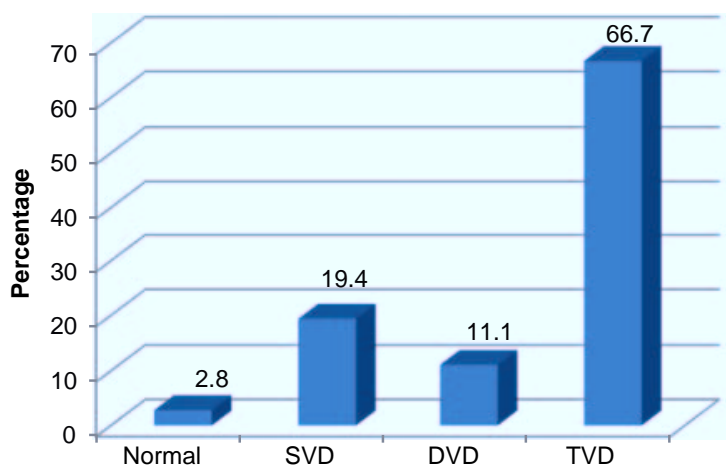

Fig.-1: Status of major coronary arteries

The angiographic findings showed that nearly threequarters $(72.2 \%)$ of the patients had LM and/or TVD (Table V). 
Table-V

Stratification of patients by LM/3VD $(n=36)$

\begin{tabular}{lcc}
\hline LM and/orTVD & Frequency & Percentage \\
\hline Present & 26 & 72.2 \\
Absent & 10 & 27.2 \\
\hline
\end{tabular}

7 Accuracy of ST-elevation in lead aVR in predicting LM/ 3VD in patients of NSTE- ACS:

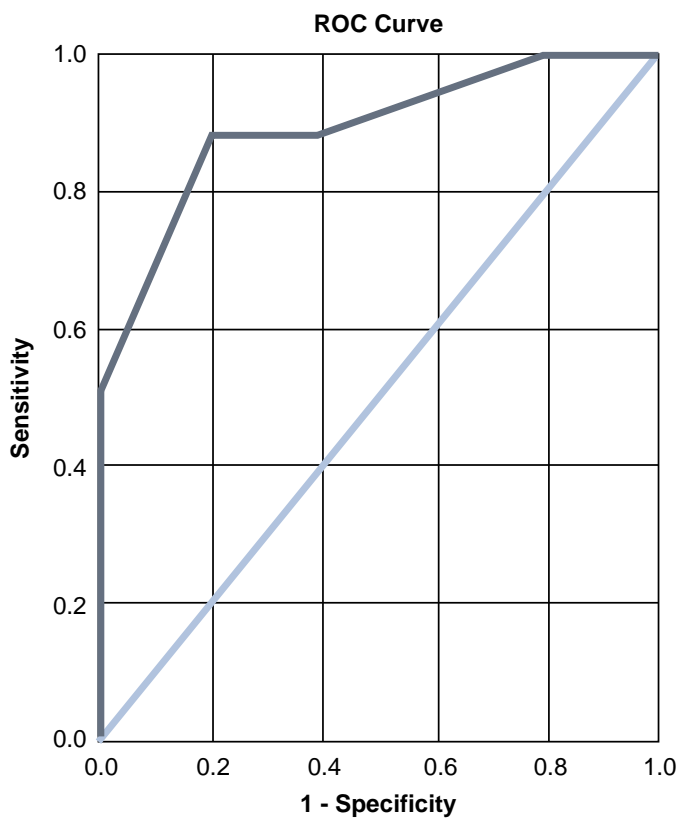

Diagonal segments are produced by ties

Fig.-2: Showing area under the ROC curve

The best cut-off value for optimum sensitivity without much compromise with specificity obtained from the table below was 0.75 with an area under the curve being $0.892(95 \%$ CI $=0.785-1.000), \mathrm{p}<0.001$ (Table VI \& VII). The area under the curve indicates that $89.2 \%$ of the LM and/or TVD could be correctly diagnosed with ST elevation e" $0.75 \mathrm{~mm}$ in lead aVR in patients with non-ST segment elevation acute coronary syndrome.

Therefore, the sensitivity of ST-elevation in lead aVR, ata cut-off value of $0.75 \mathrm{~mm}$, in diagnosing LM/3VD was, therefore, $23 / 26 ? 100=88.5 \%$ and the specificity of the test in correctly excluding those who do not have LM/3VD was $8 / 10 ? 100=80.0 \%$. The positive and negative predictive values of the test were 23/25? 100 $=92.0 \%$ and $8 / 11 \times 100=72.7 \%$ respectively. The percentages of false positive and false negatives are $2 /$ $25 ? 100=8.0$ and $3 / 11 ? \quad 100=27.3 \%$ respectively. The overall diagnostic accuracy of the test is $(23+8)$ / $(23+2+3+8) \times 100=86.1 \%$ (table VIII) .
Table VI

Area Under the Curve

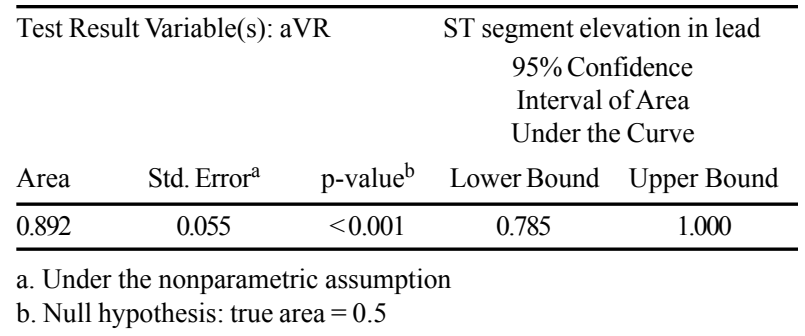

\section{Table VII}

Coordinates of the Curve

Test Result Variable(s):

ST segment elevation in lead aVR

\begin{tabular}{lcc}
\hline $\begin{array}{l}\text { Positive if Greater } \\
\text { Than or Equal To }\end{array}$ & Sensitivity & $1-$ Specificity \\
\hline-.9750 & 1.000 & 1.000 \\
.1375 & 1.000 & .800 \\
.3750 & .885 & .400 \\
.7500 & .885 & .200 \\
1.2500 & .500 & .000 \\
1.7500 & .346 & .000 \\
2.2500 & .038 & .000 \\
3.5000 & .000 & .000 \\
\hline
\end{tabular}

Table-VIII

Accuracy of ST-elevation in lead aVR in predicting $L M / 3 V D$ in patients of non-STEMI ACS

\begin{tabular}{lccc}
\hline ST-elevation in & \multicolumn{2}{c}{ LM/3VD } & Total \\
\cline { 2 - 3 } Led a VR & Present & Absent & \\
\hline$>0.75 \mathrm{~mm}$ & 23 & 02 & 25 \\
$<0.75 \mathrm{~mm}$ & 03 & 08 & 11 \\
\hline Total & 26 & 10 & 36 \\
\hline
\end{tabular}

\section{Discussion:}

In the present study, the study subjects were generally upper middle aged or elderly (72.2\%) and predominantlymale $(83.3 \%)$. More than $55 \%$ of the patients were smoker, nearly $60 \%$ were hypertensive and $47.2 \%$ diabetic. ECG showed that over two-thirds $(69.4 \%)$ of the patients had ST-elevation e" 0.75 in lead a VR. Eight patients (22.2\%) had significant LM disease. More than

$90 \%$ had significant stenosis in LAD, 77.8\% significant stenosis in LCx and 72.2\% significant stenosis in RCA. 
Two-thirds $(66.7 \%)$ of the patients had triple vessel disease (TVD), 11.1\% double vessel disease (DVD), $19.4 \%$ single vessel disease (SVD). Nearly threequarters $(72.2 \%)$ had LM and/or TVD.

The main purpose of the study was to determine the accuracy ST-segment elevation in lead aVR in predicting left main or triple vessel disease (LM/3VD) in patients with non-ST-segment elevation acute coronary syndrome. As ST-segment elevation in lead aVR is a continuous variable, a suitable cut-off for ST-elevation in lead aVR was found out for diagnosing LM and/or triple vessel disease (TVD) using ROC curve. The cut-off value was $0.75 \mathrm{~mm}$ which gave us an optimum sensitivity of $88.5 \%$ and a specificity of $80 \%$ with an area under the curve being $0.892(95 \% \mathrm{CI}=0.785-1.000)$, $\mathrm{p}<0.001$. The area under the curve demonstrated that $89.2 \%$ of the LM and/or TVD were correctly diagnosed with ST elevation e" $0.75 \mathrm{~mm}$ in lead aVR in patients with non-ST segment elevation acute coronary syndrome. The positive predictive value was commendably high (92\%) and negative predictive value was no less (72.7\%) with an overall diagnostic accuracy of $86 \%$.

Hussien and associates $(2011)^{1}$ in a similar study demonstrated that ST-segment elevation in lead aVR $\geq 0.5$ $\mathrm{mm}$ had good sensitivity (77\%) but moderate specificity (65\%) and PPV (64\%) and NPV (78\%) in diagnosing left main or triple vessel disease and concluded that this non-invasive tool could be used as a predictor of left main or three vessel disease in patients with nonST segment elevation acute coronary syndrome before angiogram for immediate patient care. These findings are consistent with the findings of the present study.

Several studies have examined the significance of STsegment elevation in lead aVR on the admission ECG in non ST-segment elevation ACS (NSTE-ACS). 2,5,6,7

Barrabes et al. 20032 examined the association between ST-segment shift in lead aVR and in-hospital mortality in 775 patients with a non-ST-segment elevation myocardial infarction (NSTEMI) and found that the rates of in-hospital mortality were $1.3 \%$ in patients without ST-segment elevation in lead aVR, $8.6 \%$ in patients with $0.05 \mathrm{mV}$ to $0.1 \mathrm{mV}$ of ST-segment elevation in lead aVR, and $19.4 \%$ in patients with ST-segment elevation e" 0.1 $\mathrm{mV}$ in lead aVR. After adjusting for clinical variables, the odds ratios (ORs) for in-hospital mortality in the last 2 groups were 4.2 (95\% CI: 1.5-12.2) and 6.6 (95\%

2.5-17.6), respectively. In 437 patients who underwent coronary arteriography within 6 months of the onset of symptoms, the prevalence of LM or 3-vessel disease among the 3 groups was $22.0 \%, 42.6 \%$, and $66.3 \%$, respectively. From the findings it appear that ST-segment elevation in lead aVR in NSTEMI is an independent predictor increased in-hospital mortality probably because of severe coronary artery disease. The findings also suggest that the greater the degree of ST-elevation inlead aVR in non-STE-ACS, the higher is the chance of death due to sever coronary artery disease. As the present study was a cross-sectional study, it did not have scope of including mortality data.

As acute occlusion of the left main (LM) artery causes life-threatening hemodynamic deterioration and malignant arrhythmias, resulting in an adverse outcome, a rapid diagnosis and subsequent urgent revascularization with percutaneous coronary intervention (PCI) or coronary bypass surgery is very important in this subset of patients. The 12-lead electrocardiogram (ECG) is a crucial tool in the diagnosis and risk stratification of acute coronary syndrome (ACS). Unlike other 11 leads, lead aVR has been long neglected until recent years.

Recent investigations have shown that an analysis of STsegment shift in lead aVR provides useful information on the coronary angiographic anatomy and risk stratification in ACS. ST-segment elevation in lead aVR can be caused by transmural ischemia in the basal part of the interventricular septum caused by impaired coronary blood flow of the first major branch originating from the left anterior descending coronary artery, ${ }^{8}$ transmural ischemia in the right ventricular outflow tract caused by impaired coronary blood flow of the large conal branch originating from the right coronary artery; ${ }^{9}$ and reciprocal changes opposite to ischemic or nonischemic ST-segment depression in the lateral limb and precordial leads10. On the other hand, ST-segment depression in lead aVR can be caused by transmural ischemia in the inferolateral and apical regions. It has been recently shown that an analysis of $\mathrm{T}$ wave in lead aVR also provides useful prognostic information in the general population and patients with prior myocardial infarction. Cardiologists should, therefore, pay more attention to the tracing of lead aVR when interpreting the 12-lead ECG in clinical practice. ${ }^{4}$

\section{Conclusion:}

From the findings of the study it can be concluded that ST- segment elevation $\geq 0.75 \mathrm{~mm}$ in lead aVR in patients of non-ST segment elevation acute coronary syndrome had optimum sensitivity and specificity with an appreciably high overall diagnostic accuracy. The ST- 
segment elevation $\geq 0.75 \mathrm{~mm}$ in lead aVR in patients with non-STE-ACS can differentiate LM and/or triple vessel disease with fair degree of accuracy. So this noninvasivetool could be used as a predictor of left main or three vessel disease in patients with non-ST segment elevation acute coronary syndrome before angiogram for immediate patient management.

We have some limitations in our study

1. The study was conducted to one selected specialized hospital due to shortage of time, limited financial support and administrative inconveniences. Due to small amount of sample size was taken. Therefore the small sample might not represent the true picture of all people of the whole country.

2. Inherence weakness of the study design-cross sectional. The study population was selected purposively from one hospital. So, it does not necessarily represent situations prevailing in other part of the country.

3. Due to time constrained, the study involved only 36 respondents. So the results may not coincide with large scale survey.

\section{Conflict of Interest}

Authors has no conflict of interest.

\section{Acknowladgement}

This study was a postgraduate thesis. The authors would like to thank all of the patients who enrolled in this study and staff of the Department of Cardiology, University Cardiac Center, Bangabandhu Sheikh Mujib Medcal University, Dhaka, Bangladesh. Financial support was obtained from the Research Council of the Bangabandhu Sheikh Mujib Medical University, Dhaka, Bangladesh.

\section{References:}

1. Hussien A, Battah A, Ashraf M, El-Deen TZ, Electrocardiography as a predictor of left main or three-vessel disease in patients with non-ST segment elevation acute coronary syndrome. The Egyptian Heart Journal 2011; 63:103-7.

2. Barrabés JA, Figueras J, Moure C, Cortadellas J, Soler-Soler J, Prognostic value of lead aVR in patients with a first non-ST-segment elevation acute myocardial infarction. Circulation 2003; 108:814-9.

3. Azam MG, Al-Baker SME, Rahman Z, Rahman MM, Ahmed CM, Benarjee SK. et al. Correlation of the ECG changes (ST segment depression with or without $\mathrm{T}$ wave inversion in lateral leads 1 , avl, V4- V6) with coronary angiographic findings in ACS patients. University Heart Journal 2013; 9(1):9-12.

4. Tamura A, Significance of lead aVR in acute coronary syndrome. World J Cardiol 2014;6(7):630-7.

5. Yan AT, Yan RT, Kennelly BM, Anderson FA, Budaj A, LópezSendón J, Relationship of ST elevation in lead aVR with angiographic findings and outcome in non-ST elevation acute coronary syndromes. Am Heart J 2007; 154:71-8.

6. Taglieri N, Marzocchi A, Saia F, Marrozzini C, Palmerini T, Ortolani P, Short- and long-term prognostic significance of STsegment elevation in lead aVR in patients with non-ST-segment elevation acute coronary syndrome. Am J Cardiol 2011;108: $21-8$

7. Kosuge M, Kimura K, Ishikawa T, Ebina T, Shimizu T, Hibi $\mathrm{K}$, Predictors of left main or three- vessel disease in patients who have acute coronary syndromes with non-ST-segment elevation. Am J Cardiol 2005; 95:1366-9.

8. Gorgels AP, Engelen DJ, Wellens HJ, Lead aVR, a mostly ignored but very valuable lead in clinical electrocardiography. J Am Coll Cardiol 2001;38:1355-6

9. Yamaji H, Iwasaki K, Kusachi S, Murakami T, Hirami R, Hamamoto H, Prediction of acute left main coronary artery obstruction by 12-lead electrocardiography. ST segment elevation in lead aVR with less ST segment elevation in lead V(1). J Am Coll Cardiol 2001; 38:1348-54.

10. Nikus KC, Eskola MJ, Electrocardiogram patterns in acute left main coronary artery occlusion. J Electrocardiol 2008;41:626-9. 